\title{
Enhancement of Histone Deacetylase Inhibitor Sensitivity in Combination with Cyclin-Dependent Kinase Inhibition for the Treatment of Oral Squamous Cell Carcinoma
}

\author{
Borui Zhao Zhi Huang $^{\mathrm{a}}$ Zhongxiang Qin ${ }^{\mathrm{a}} \quad$ Yongtao Lia Tianqi Wang ${ }^{\mathrm{a}}$ \\ Linchuan Wang ${ }^{\mathrm{b}, \mathrm{c}}$ Wei Zhou ${ }^{\mathrm{a}}$ Chenhua Yu $\mathrm{Xin}^{\mathrm{a}}$ Wang $^{\mathrm{a}}$ \\ Shengyong Yang ${ }^{d}$ Yan Fan $^{\mathrm{a}, \mathrm{e}}$ Rong Xianga,e \\ aDepartment of Medicinal Chemistry, School of Medicine, Nankai University, Tianjin, China, \\ bDepartment of General Dentistry University of Rochester Eastman Insititute for Oral Health, Rochester, \\ NY, USA, cPeking University School and Hospital of Stomatology, Beijing, China, 'State Key Laboratory \\ of Biotherapy and Cancer Center, West China Hospital, Sichuan University and Collaborative Innovation \\ Center for Biotherapy, Chengdu, Sichuan, China, ${ }^{\mathrm{e}} 2011$ Project Collaborative Innovation Center for \\ Biotherapy of Ministry of Education, Tianjin, China
}

\section{Key Words}

OSCC $・$ Inhibitor $\cdot \mathrm{HDAC} \cdot \mathrm{CDK}$

\begin{abstract}
Background/Aims: Previous research has indicated that the currently available histone deacetylase inhibitors (HDACis) are not effective as monotherapies against oral squamous cell carcinoma (OSCC). However, HDACis act synergistically with other therapeutic agents to exert significant antitumor activities. Thus, a strategy to develop chemotherapeutic agents by combining several active groups based on histone deacetylase (HDAC) into a single molecule as a conjugate that modulates multiple cellular pathways may be useful for the treatment of OSCC. Methods: The novel inhibitor Roxyl-ZR was prepared by organic synthesis and its anticancer effects on OSCC were investigated by cell metabolism $(n=5)$, colony formation $(n=3)$, cell cycle $(n=3)$, cell apoptosis $(n=3)$, wound healing $(n=3)$, transwell migration $(\mathrm{n}=3)$, and 5-bromo-2'-deoxyuridine staining $(\mathrm{n}=3)$ assays in vitro and in in vivo xenograft mice models (4 mice/group for subcutaneous xenograft and 3 mice/group for orthotopic xenograft). The abundance of $\mathrm{Ki} 67, \mathrm{Bcl}-2$, and $\mathrm{p}-\mathrm{STAT} 3$ was detected by immunohistochemistry staining $(n=4)$. Apoptotic cells in the tumor tissues of mice were detected by terminal deoxynucleotidyl transferase dUTP nickend labeling assay $(n=3)$. The abundance of related

B. Zhao and Z. Huang contributed equally to this work.

\begin{tabular}{ll}
\hline Yan Fan & Department of Medicinal Chemistry, School of Medicine, Nankai University \\
and Rong Xiang & 94 Weijin Road, Tianjin, 300071 (China) \\
& E-Mail yanfan@nankai.edu.cn; rxiang@nankai.edu.cn
\end{tabular}
\end{abstract}




\section{Cellular Physiology Cell Physiol Biochem 2019;53:141-156 \\ \begin{tabular}{ll|l} 
and Biochemistry $\begin{array}{l}\text { DOl: 10.33594/000000126 } \\
\text { Published online: } 26 \text { June 2019 }\end{array}$ & $\begin{array}{l}\text { O } 2019 \text { The Author(s). Published by } \\
\text { Cell Physiol Biochem Press GmbH\&Co. KG }\end{array}$
\end{tabular} \\ Zhao et al.: Novel CDK-HDAC Inhibitor for the Treatment of Oral Cancer}

proteins levels were evaluated by western blot $(n=3)$. E-cadherin expression was detected by an immunofluorescence assay $(n=3)$. Results: Compared with the approved $\mathrm{HDACi}$, conjugated Roxyl-ZR exhibited significantly higher antitumor effects in OSCC cells. Roxyl-ZR suppressed OSCC cell proliferation by inducing the reduction of $S$ phase and inducing caspase-dependent apoptosis by down-regulating Bcl-2 expression. Moreover, Roxyl-ZR attenuated the epithelialmesenchymal transition, which is closely associated with migration and invasion. In addition, Roxyl-ZR inhibited OSCC xenograft mice models and showed low toxicity. The mechanism underlying the Roxyl-ZR-enhanced sensitivity to HDACi may be attributed to the inhibition of key regulators of JAK1-STAT3 signaling pathway. Conclusion: HDAC-cyclin-dependent kinase conjugates represent a novel approach to the development of OSCC treatment. Our findings may open a new avenue for the development of novel inhibitors for the treatment of OSCC.

(C) 2019 The Author(s). Published by

Cell Physiol Biochem Press GmbH\&Co. KG

\section{Introduction}

OSCC is the most prevalent oral tumor, grows rapidly, and is highly invasive, resulting in a poor prognosis and recurrences associated with a 5-year survival rate of less than $50 \%$ [1, 2]. A poor prognosis and the tendency for relapses occur in patients with aggressive solid tumors, chemotherapy-resistant recurrences, or metastasis after treatment [3]. Furthermore, conventional chemotherapy for the treatment of OSCC is limited by its frequent recurrences and secondary neoplastic lesions, which are followed by drug resistance [4]. Thus, novel therapeutic inhibitors for the treatment of OSCC treatment are urgently needed.

OSCC is a highly complex multigenetic disease involving multiple cross-talks among signaling networks and often requires multiple therapeutic interventions. The use of combination therapy with multiple drugs that work through different mechanisms is a proven therapeutic strategy for the management of cancer [5, 6]. Small molecule inhibitors against several pathway targets are undergoing clinical evaluation as anti-cancer agents [7, 8].

Epigenetic modifications to the histones of chromatin play a predominant role in the control of transcription and regulation of genes [9]. Histone deacetylase inhibitor (HDACi) induces cell cycle arrest, apoptosis, differentiation and simultaneously inhibits angiogenesis, invasiveness, and metastasis [10-12]. As reported previously, overexpression of histone deacetylase (HDAC) is associated with the development of OSCC; thus, HDACis could be considered potential anticancer agents for the treatment of OSCC [13]. Despite the satisfactory potency of HDACis, when used as single-agent therapies, they do not produce the objective response, and relatively high doses are required to achieve full inhibition of the target, which then leads to a greater number of side effects. Studies of experimental and clinical data have demonstrated that the currently available HDACis are not adequate as monotherapies against OSCC [13, 14]. However, HDACis exert significant antitumor activity in combination therapies. The synergistic action between HDACis and other therapeutic agents could achieve higher antiproliferative effects in OSCC therapy [13-16].

Accumulating data suggest that the synergistic effects of HDACis in conjunction with other antitumor agents could be used as a strategy to design multifunctional inhibitors that simultaneously interact with multiple targets in the treatment of OSCC. Cyclin-dependent kinases (CDKs) are a good starting point for the design of multivalent ligands. CDKs with corresponding cyclins regulate the progression of mitosis [17]. Since the importance of the activity of CDKs in cancer progression is already known, CDKs inhibitors have emerged as attractive therapeutic strategies [18]. Our recent studies focusing on combining the CDKs pharmacophore with HDAC or another target revealed enhanced antitumor activity $[19,20]$. Based on a synergistic experiment in OSCC in vitro and in vivo, we found that vorinostat, an HDACi, worked synergistically with abemaciclib, a CDK4/6 inhibitor, with a combination index (CI) of < 1 (Supplementary Fig. S1 and S2 - for all supplemental material see www. cellphysiolbiochem.com). Thus, a single molecule able to inhibit CDKs and HDAC may enhance the sensitivity of HDACis and would be helpful for the treatment of OSCC. 


\section{Cellular Physiology Cell Physiol Biochem 2019;53:141-156 \\ \begin{tabular}{cc|c} 
DOl: $10.33594 / 000000126$ & O) 2019 The Author(s). Published by \\
Cell Physiol Biochem Press GmbH $\&$ Bico
\end{tabular} \\ Zhao et al.: Novel CDK-HDAC Inhibitor for the Treatment of Oral Cancer}

Therefore, to address the need for more effective drugs based on HDACis for OSCC, we designed Roxyl-ZR, a novel small inhibitor that conjugates CDK4 by organic syntheses. We then evaluated the therapeutic efficacy and mechanism of Roxyl-ZR in OSCC in vitro and in vivo.

\section{Materials and Methods}

\section{Cell culture}

Human OSCC cell lines were kindly provided by Professor Xudong Wang of the Department of Maxillofacial and E.N.T Oncology at Tianjin Medical University Cancer Institute and Hospital, Tianjin, China. UM1 and SCC-25 cells were maintained in Dulbecco's modified Eagle's medium/F12 medium (Biological Industries (BI), Cromwell, CT). TSCCA cells were maintained in minimum essential medium (Corning, Corning, NY). Tca-8113 and Tb3.1 cells were maintained in 1640 medium (BI). All cell lines were supplemented with $10 \%$ fetal bovine serum (FBS; BI) and $1 \%$ penicillin/streptomycin (BI) at $37^{\circ} \mathrm{C}$ in atmosphere of $5 \% \mathrm{CO}_{2}$.

Half maximal inhibitory concentration $\left(I C_{50}\right)$ calculation and cell metabolism assay

Cell metabolism assays were conducted as described previously using a Cell Counting Kit-8 (CCK-8) assay (\#CK04, Dojindo, Kumamoto, Japan) [19]. Briefly, $\mathrm{IC}_{50}$ values were quantified after $72 \mathrm{~h}$. For the timedependent cell metabolism assay, cell metabolism was measured at $0,12,24,36$, and $48 \mathrm{~h}$. The absorbance was measured at $450 \mathrm{~nm}$ using a microplate enzyme-linked immunosorbent assay (ELISA) reader.

\section{Colony formation assay}

UM1 and TSCCA cells $\left(4 \times 10^{3}\right.$ cells/well) were seeded in 24-well plates and cultured with different concentrations of inhibitors or vehicle. Cells were treated with inhibitors or vehicle for 5 days. After 5 days, cells were fixed with $20 \%$ methanol, stained with $0.5 \%$ crystal violet, and photographed at $20 \times$ magnification under a light microscope (Olympus, Tokyo, Japan). Cells were counted using ImageJ software (National Institutes of Health, Bethesda, MD). The results are presented in a bar graph.

\section{5-Bromo-2'-deoxyuridine staining (BrdU)}

BrdU staining was conducted according to the manufacturer's instructions (APC BrdU Flow Kit, \#519000019AK, BD Biosciences, Franklin Lakes, NJ). UM1 and TSCCA cells $\left(1 \times 10^{5}\right)$ were seeded in 6-well plates and treated with Roxyl-ZR, vorinostat, abemaciclib, or vehicle for $48 \mathrm{~h}$. Data were analyzed using BD FACSDiva $^{\mathrm{TM}}$ version 6.1.3 and BD CellQuest ${ }^{\mathrm{TM}}$ PRO software (BD Biosciences).

\section{Flow cytometry}

Cell cycle and cell apoptosis assays (FITC Annexin V Apoptosis Detection Kit, \#556547, BD Biosciences) were conducted as described previously [6]. Briefly, cells were treated for $36 \mathrm{~h}$ in the cell cycle assay and for $48 \mathrm{~h}$ in the cell apoptosis assay. Data were analyzed using BD FACSDiva ${ }^{\mathrm{TM}}$ version 6.1.3 and BD CellQuest ${ }^{\mathrm{TM}}$ Pro software.

\section{Wound healing assay}

UM1 and TSCCA cells were seeded in 6-well plates until fully confluent and then wounded by scratching

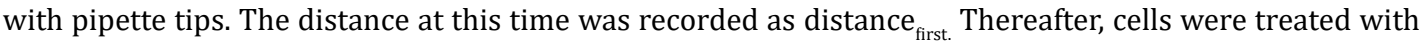
inhibitors with different concentrations or vehicle for 12 and $24 \mathrm{~h}$, respectively. The distance at this time was recorded as distance final. $_{\text {. }}$ The migrated distance was measured using ImageJ version 1.47 software and photographed under a light microscope (Olympus). The migrating distance was calculated using the following formula: migrating distance $=$ distance $_{\text {first }-}$ distance $_{\text {final }}$.

Transwell migration assay

Transwell chambers (\#MCEP24H48, Millicell Hanging) were used in accordance with the manufacturer's instructions. UM1 or TSCCA cell suspensions $\left(200 \mu \mathrm{L} ; 5 \times 10^{4}\right.$ cells/well) were placed in the top chamber and treated with different concentrations of inhibitors or vehicle with 1\% FBS for $16 \mathrm{~h}$. 


\section{Cellular Physiology Cell Physiol Biochem 2019;53:141-156 \\ \begin{tabular}{c|c|c|} 
Dol: 10.33594/000000126 & O 2019 The Author(s). Published by \\
Cell Physiol Biochem Press GmbHerco
\end{tabular} \\ Zhao et al.: Novel CDK-HDAC Inhibitor for the Treatment of Oral Cancer}

Medium $(500 \mu \mathrm{L})$ with $10 \%$ FBS was added to the lower chamber. After $16 \mathrm{~h}$, non-migrated cells were wiped away with cotton swabs, and migrated cells were fixed with $20 \%$ methanol, stained with $0.5 \%$ crystal violet, and photographed at 20× magnification under a light microscope (Olympus). Cells were counted using Image J software. The results are presented in a bar graph.

\section{Western blot assay}

After treatment with inhibitors or vehicle for $48 \mathrm{~h}$, UM1 and TSCCA cells were washed three times with phosphate-buffered saline (PBS) and lysed in RIPA-buffer. The preparation of total cell extracts and immunoblotting with appropriate antibodies were performed as previously described [21]. The following primary antibodies were used: CDK4 (1:1000, sc-23896, Santa Cruz, CA), caspase-3 (1:500, sc-7148, Santa Cruz), STAT3 (1:1000, sc-8019, Santa Cruz), phosphorylated (p)-STAT3 (1:1000, sc-8059, Santa Cruz), $\beta$-actin, (1:1000, sc-47778, Santa Cruz), cyclin D1 (1:1000, \#2978S, Cell Signaling Technology (CST), Danvers, MA), Rb (1:1000, \#9309S, CST), p-Rb (1:2000, \#8516S, CST), ac-H3 (1:1000, \#9649S, CST), N-cadherin (1:1000, \#13116S, CST), vimentin (1:1000, \#5741S, CST), Bcl-2 (1:1000, \#51-6511GR, BD Biosciences), E-cadherin (1:1000, \#610182, BD Biosciences), Ki67 (1:1000, \#GR259204-2, Abcam, Cambridge, UK), matrix metalloproteinase-2 (MMP-2; 1:1000, \#66366-1-lg, Proteintech, Rosemont, IL), and MMP-9 (1:500, \#10375-2-AP, Proteintech).

Protein density was quantified using ImageJ version 1.60 software. All lanes were normalized by dividing by the density of vehicle group.

\section{Immunofluorescence assay}

Circular cover glasses were rinsed with 75\% alcohol and PBS and placed into 24-well plates. Thereafter, UM1 and TSCCA cells $\left(5 \times 10^{4}\right)$ were seeded on the cover glasses and treated with inhibitors or vehicle for $48 \mathrm{~h}$. Cells were harvested and fixed with $4 \%$ paraformaldehyde for $10 \mathrm{~min}$ at room temperature. Cells were blocked for $1 \mathrm{~h}$ with PBS containing 5\% goat serum at room temperature and then incubated with primary antibodies of E-cadherin $(1: 200)$ at $4^{\circ} \mathrm{C}$ overnight. Cells were subsequently incubated with Alexa 488-conjugated anti-rabbit or anti-mouse use IgG for $1 \mathrm{~h}$ at room temperature. Nuclei were counterstained with a DAPI karyotyping kit. Slides were photographed using an FV-1000 laser scanning confocal biological microscope (Olympus).

\section{Luciferase transfection and selection of stable cell lines}

UM1 cells was infected with lentivirus carrying pLv-EF1 $\alpha$-luciferase-IRES-Neo (pLV-Luc) or empty vector, and then persistently selected by $1.5 \mathrm{mg} / \mathrm{mL}$ G418 for 2 weeks to generate a stable cell line with luciferase expression (UM1-luc).

\section{Animal models}

The experimental procedures of the animal study were approved by the Animal Care and Use Committee at Nankai University (Nankai, China). Male Balb/c nude mice aged 6-8 weeks old were housed in a specific pathogen-free animal facility. To implement subcutaneous human OSCC tumors, $1.5 \times 10^{6}$ UM1 cells were dispensed in $100 \mu \mathrm{L}$ of $1 / 1$ mixture of medium/Matrigel (BD Biosciences) and injected into the right flanks of the mice. When tumors reached a volume of $100 \mathrm{~mm}^{3}$, mice were randomly divided into six groups (4 mice/group) and received oral administration of different doses of inhibitors or vehicle once per day. Tumor volume $(V)$ was calculated by measuring the length $(L)$ and width $(W)$ of the tumor with calipers using the following formula: $V=L \times W^{2} / 2$. To establish orthotopic OSCC mice models, $1 \times 10^{6}$ UM1-luc cells were injected into the floor of the mouth and mice were randomly divided into six groups ( 3 mice/group). Different doses of inhibitors or vehicle were administered orally once a day. The body weights of the mice were also monitored every 3 days. Oral gavage was continued for 21 days.

\section{Hematoxylin and eosin (HE) staining and immunohistochemistry staining}

HE staining was performed as previously described [6]. The primary antibodies included Ki67 (1:200), Bcl-2 (1:200), and p-STAT3 (1:200) as described. The H-score for each protein incorporates staining intensity (negative, weak, moderate and strong were graded as 0, 1, 2, 3 separately) multiplied by the percentage of positive cells based on the images observed under a light microscope (Olympus). 


\section{Cellular Physiology Cell Physiol Biochem 2019;53:141-156 \\ \begin{tabular}{ll|l} 
DOl: $10.33594 / 000000126$ & O 2019 The Author(s). Published by \\
Cell Physiol Biochem Press GmbH $\&$ Bioco
\end{tabular} \\ Zhao et al.: Novel CDK-HDAC Inhibitor for the Treatment of Oral Cancer}

Terminal deoxynucleotidyl transferase dUTP nick end labeling (TUNEL) assay

Apoptotic cells in the tumor tissues of mice were detected by the DeadEnd ${ }^{\mathrm{TM}}$ Colorimetric TUNEL system (\#G7130 Promega, Madison, WI) according to the experimental protocols. Nuclei were counterstained with a DAPI karyotyping kit. Positive cells were visualized using an FV-1000 laser scanning confocal biological microscope.

\section{Bioluminescent imaging in vivo}

The orthotopic xenograft tumor mice of each group were anesthetized with $4 \%$ chloral hydrate, and $200 \mu \mathrm{L}$ D-luciferin $(15 \mathrm{mg} / \mathrm{mL}$, Sinochrome, \#bc219-01) was injected intraperitoneally for 10 min before imaging. Mice were set on the imaging platform and photographed with bioluminescent imaging (BLI) using an IVIS50 imaging system (IVIS Lumina II, Life Science, Carlsbad, CA) once per week. Regions of interest were circled around the tumors and measured using Igor Pro 4.09A imaging software (WaveMetrics, Lake Oswego, OR) as photons $/ \mathrm{s} / \mathrm{cm}^{2} / \mathrm{sr}$.

Acute toxicity assay

Balb/c mice (6-8 weeks) were treated with Roxyl-ZR $80 \mathrm{mg} / \mathrm{kg}$ or vehicle for $48 \mathrm{~h}$. Organs were excised after the mice were sacrificed. Organ toxicity analysis was conducted by HE staining. For routine examinations of blood, mice were treated with Roxyl-ZR $80 \mathrm{mg} / \mathrm{kg}$ or vehicle once every 2 days, up to a total of 4 administrations, and then sacrificed. Blood was examined using a hematology analyzer (Celltac E, Nihon Kohden, Tokyo, Japan).

\section{Synergic effect assay}

The combination index (CI) value was calculated as follows:

$\mathrm{CI}=\left([\mathrm{cA}+\mathrm{cB}]-\left[\mathrm{cA}{ }^{*} \mathrm{cB}\right]\right) / \mathrm{cAB}$

Where $\mathrm{CA}$ is the inhibitory rate of compound $\mathrm{A}, \mathrm{CB}$ is the inhibitory rate of compound $\mathrm{B}$, and $\mathrm{CAB}$ is the inhibitory rate of combination treatment with compounds A and B. Synergy is defined as $\mathrm{CI}$ lower than 1.0, and antagonism was defined as CI significantly higher than 1.0.

\section{Chemistry and molecular docking}

The chemical information of the compounds, enzyme inhibition assays, and docking methods are provided in the Supplemental Material.

\section{Statistical analysis}

Data are presented as the mean \pm standard deviation. All statistical analyses were performed using GraphPad Prism 5 software (GraphPad Software Inc., La Jolla, CA). The unpaired Student's $t$-test was used for the comparisons between two different groups. A p-value less than 0.05 was considered significant. * $p$ $<0.05,{ }^{* *} \mathrm{p}<0.005,{ }^{* * *} \mathrm{p}<0.001$.

\section{Results}

\section{Multitarget small inhibitor of HDAC and CDK4}

Based on the synergistic experimental results, in which the mean CI values of vorinostat (an HDACi) and abemaciclib (a CDK4 inhibitor) in vitro and in vivo were less than 1 (Supplementary Fig. S1 and S2), we aimed to design a conjugate with essential pharmacophoric elements targeting HDAC and CDK. This led to the discovery of compound Roxyl-ZR based on vorinostat and abemaciclib (Fig. 1A). The inhibitory potency against CDKs and HDAC was evaluated by in vitro enzyme inhibition assays provided by Eurofins Scientific (Luxembourg) and BPS Bioscience Inc. (San Diego, CA), respectively (Table 1). Roxyl-ZR was confirmed as an excellent inhibitor of CDK4 and HDAC1, with $\mathrm{IC}_{50}$ values of 1.2 and $17 \mathrm{nM}$, respectively.

Molecular docking studies were performed to identify the molecular target of RoxylZR. Kinase assay results showed that Roxyl-ZR showed a similar level of CDK4 inhibition to abemaciclib. As shown in Fig. 1B, Roxyl-ZR could tightly bind to the ATP-binding site of 


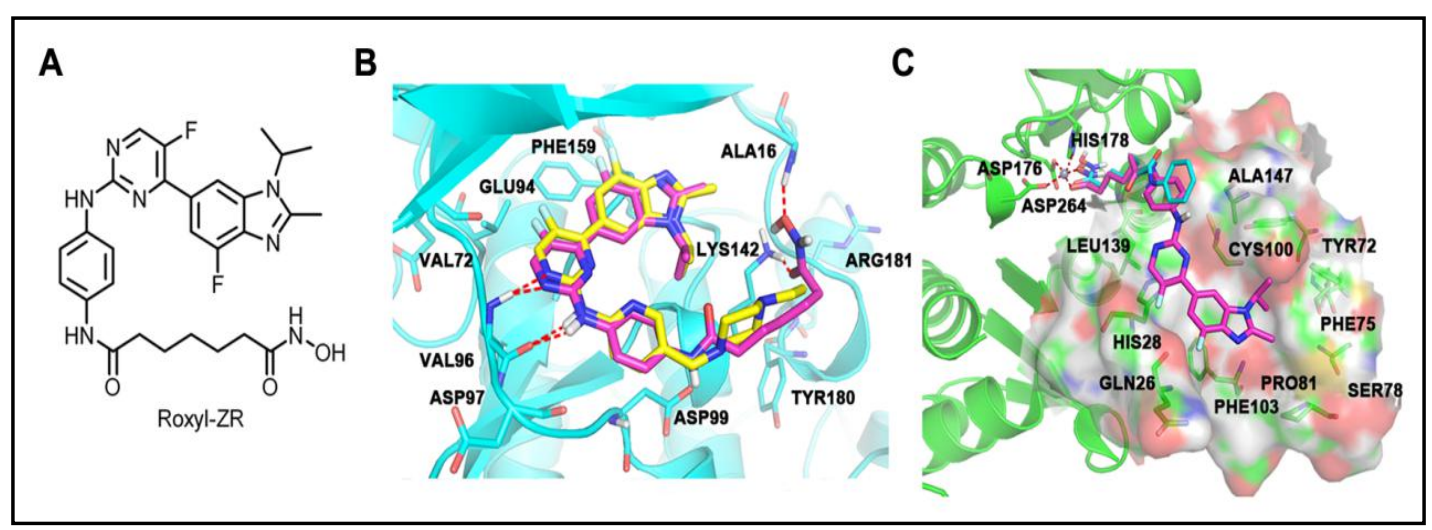

Fig. 1. Roxyl-ZR, a novel hybrid compound targeting CDK4 and HDAC1. (A) Structure of Roxyl-ZR. (B) Representation of the predicted binding modes of Roxyl-ZR (purple) and abemaciclib (yellow) in the ATP pocket of CDK4. Dashed lines indicate the H-bond interaction between compounds and CDK4. (C) Predicted binding mode of compound Roxyl-ZR (purple) and vorinostat (cyan) with HDAC1.

CDK4 in a binding mode similar to that of abemaciclib. As shown in Fig. 1C, the hydroxamic acid of Roxyl-ZR could insert into the catalytic outer tunnel of HDAC1 and the group also chelated a zinc ion, which is important for the catalytic process of HDAC1 and may explain the binding affinity of compound Roxyl-ZR, which is the same as vorinostat. These results indicated that Roxyl-ZR is a dual-function inhibitor, thus demonstrating the effectiveness of our multitarget design strategy.

The compound was next evaluated in a dose-response study against five OSCC cell lines UM1, TSCCA, SCC-25, Tca-8113, and Tb3.1 using vorinostat, abemaciclib, or a vehicle as control groups by CCK- 8 assay. The results showed Roxyl-ZR exhibited significantly higher activity with an $\mathrm{IC}_{50}$ value around $200 \mathrm{nM}$, while vorinostat or abemaciclib had IC $_{50}$ values were mostly $>1$ $\mu \mathrm{M}$ (Table 2). Since most normal proliferating cells used CDK4/6 to proliferate, Roxyl-ZR, similarly to abemaciclib as a CDK inhibitor, exhibited no tumor specificity against HaCaT cells, which are immortalized epithelial cells. However, tumor cells grow much more rapidly than do normal cells, which were sensitive to treatment with CDK inhibitors. In addition, acute general toxicity analysis indicated that Roxyl-ZR was non-toxic in mice when compared with the vehicle group (Supplementary Fig. S3).

\section{Roxyl-ZR inhibited OSCC metabolism and clonogenicity in vitro}

Because UM1 and TSCCA cells are high- and low-invasive cells, respectively, we chose these two cell lines for subsequent biological studies. We selected $100 \mathrm{nM}$ and $200 \mathrm{nM}$ as the respective concentrations, and cell metabolism was examined after treatment with Roxyl-ZR or vehicle at $0,12,24,36$, and $48 \mathrm{~h}$. The results indicated that Roxyl-ZR effectively inhibited cell metabolism in dose- and time-dependent manner compared with the other groups (Fig. 2A). Colony formation assays confirmed that Roxyl-ZR groups significantly reduced clonogenicity compared with the other treatments (Fig. 2B). BrdU assay revealed that Roxyl- 


\section{Cellular Physiology Cell Physiol Biochem 2019;53:141-156 \\ \begin{tabular}{ll|l} 
and Biochemistry $\begin{array}{l}\text { DOl: 10.33594/000000126 } \\
\text { Published online: } 26 \text { June 2019 }\end{array}$ & $\begin{array}{l}\text { O } 2019 \text { The Author(s). Published by } \\
\text { Cell Physiol Biochem Press GmbH\&Co. KG }\end{array}$ \\
\cline { 2 - 3 } &
\end{tabular} \\ Zhao et al.: Novel CDK-HDAC Inhibitor for the Treatment of Oral Cancer}

ZR significantly inhibited chromatin replication in cell cycle S phase of UM1 and TSCCA cells, and consequently decreased the ratio of $\mathrm{BrdU}^{+}$cells compared with the other groups (Fig. 2C). Collectively, these results indicated that Roxyl-ZR had greater antitumor effects on metabolism, clonogenicity, and proliferation of OSCC cells than that of control group.

\section{Roxyl-ZR inhibited OSCC migration and invasion}

OSCC can invade surrounding tissues and metastasis to other organs. It was previously confirmed that the epithelial-mesenchymal transition (EMT) is linked to OSCC progression, including cell invasion and metastasis. Previous studies have shown that vorinostat can restrain migration and invasion of several types of tumors and suppress EMT progression $[22,23]$. Wound healing and transwell assays were performed to observe the ability of RoxylZR to inhibit migration and invasion of OSCC cells in vitro. The migratory distance in RoxylZR groups was remarkably shorter than that in the other groups (Fig. 3A). The transwell assay demonstrated that after $16 \mathrm{~h}$, OSCC cells treated with Roxyl-ZR had suppressed migration compared with the other groups (Fig. 3B). Western blot assay evaluated the expression level of EMT-associated proteins treated with Roxyl-ZR, vorinostat, abemaciclib, or vehicle at $100 \mathrm{nM}$ and $200 \mathrm{nM}$. The results showed that the expression level of epithelial marker E-cadherin was up-regulated, while the mesenchymal markers $\mathrm{N}$-cadherin and vimentin were down-regulated. In addition, invasion associated marker MMP-2 and MMP9 were also down-regulated (Fig. 3C and Supplementary Fig. S4). Significant up-regulation of E-cadherin, detected by immunofluorescence analysis, also demonstrated the inhibitory activity of compound Roxyl-ZR on cell invasion (Fig. 3D). Combined with the above findings, these results verified that Roxyl-ZR could effectively inhibit the migration and invasion of OSCC cells.

Roxyl-ZR induced cell cycle arrest and apoptosis in OSCC cells via CDK and HDAC pathways

We considered whether the inhibition of cell proliferation partly resulted from cell cycle inhibition and apoptosis. FACS analysis revealed that abemaciclib treatment resulted in an accumulation of cells in the $G_{0} / G_{1}$ phase. Cells treated with vorinostat showed a degree of $\mathrm{G}_{2}$ arrest. Roxyl-ZR significantly induced $\mathrm{G}_{2}$ arrest and apoptosis at $100 \mathrm{nM}$ and $200 \mathrm{nM}$ and decreased the number of cells in the S phase (Fig. 4A and Supplementary Fig. S5). These results were consistent with those analyzed using APC-BrdU staining (Fig. 2C). Subsequently, the effect of Roxyl-ZR on cell apoptosis was conducted using annexin V/propidium iodide (AV/PI) dual staining. Compared with control groups, treatment with Roxyl-ZR markedly increased AV-positive early-stage and late-stage apoptotic cells (Fig. 4B and Supplementary Fig. S6). To determine whether Roxyl-ZR inhibited the abundance levels of cell cycleassociated proteins and down-stream signaling pathways, western blot assays indicated that Roxyl-ZR decreased the abundance of cyclin D1, p-Rb, and Ki67 dose-dependently. Meanwhile, Roxyl-ZR up-regulated CDK4 and HDAC1 associated protein ac-H3 abundance (Fig. 4C and Supplementary Fig. S7). The increased abundance of CDK4 might have resulted as compensation for the increased CDK inhibition. The effect on the abundance of Ac-Histone $\mathrm{H} 3$ via CDK4 inhibitor abemaciclib could be related to the phosphorylation of $\mathrm{Rb}[24,25]$. Roxyl-ZR also suppressed the abundance level of apoptosis associated proteins Bcl-2 and induced cleaved caspase-3 expression compared with the other groups. In a previous study, STAT3 stimulated anti-apoptotic signaling, which accounted for the limited response to HDACi treatment, and JAK1 was mainly responsible for STAT3 activation [26]. Based on the measurement of kinase activity on JAK1 (Supplementary Fig. S8), Roxyl-ZR dose-dependently decreased the abundance of p-STAT3, the downstream effector of JAK1, according to western blot analysis (Fig. 4D and Supplementary Fig. S9). Vorinostat and abemaciclib exhibited weaker effects. These results showed that the inhibition of OSCC cell growth induced by Roxyl-ZR was associated with cell cycle arrest and cell apoptosis. Inhibition of the JAK1- 


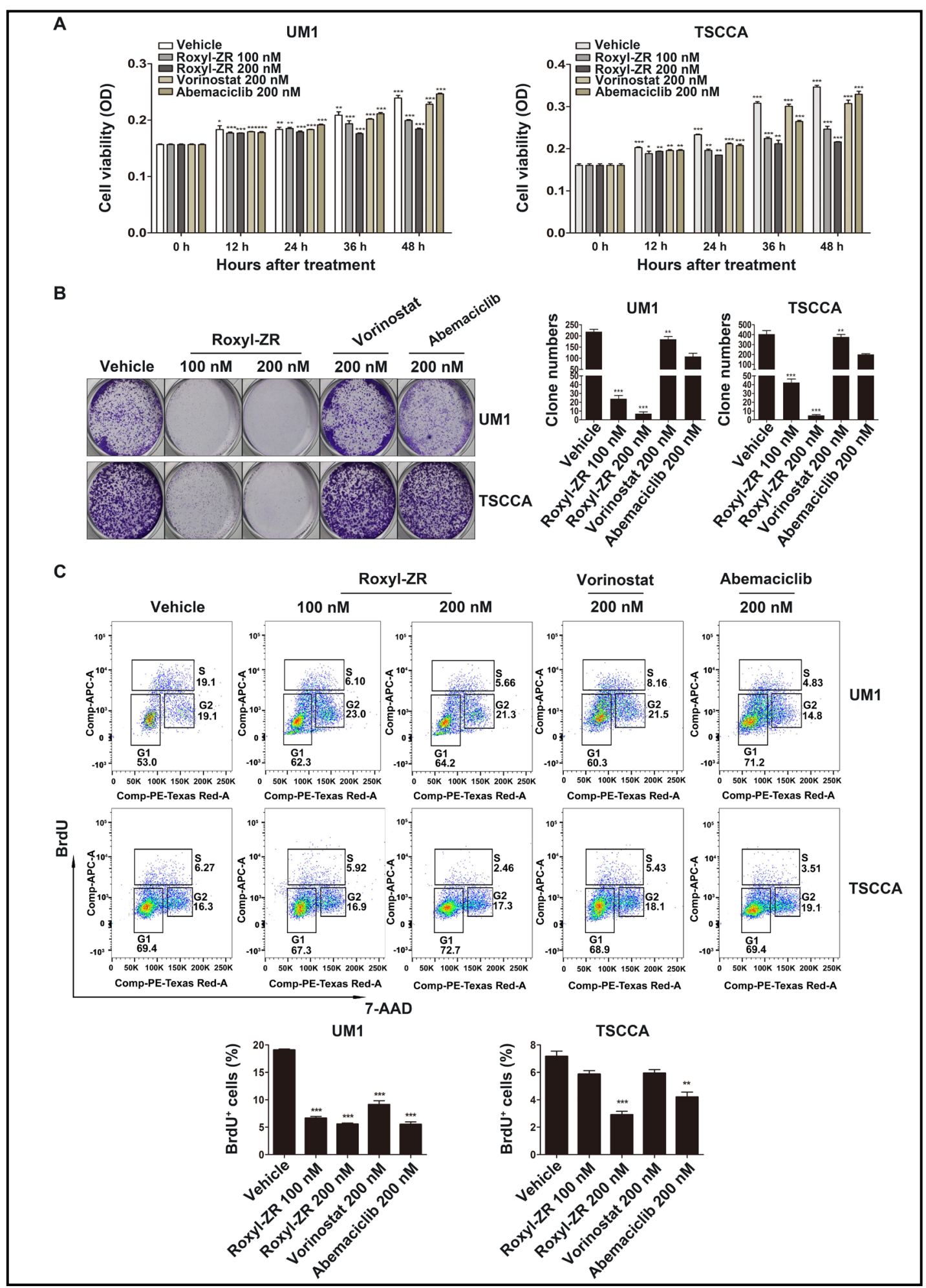

Fig. 2. Roxyl-ZR suppresses the proliferation of OSCC. (A) Metabolism of UM1 and TSCCA cells was inhibited using Roxyl-ZR in a dose- and time-dependent manner compared with the other groups, as determined by CCK-8 assay. (B) Roxyl-ZR effectively inhibited the proliferation of UM1 and TSCCA cells in 5 days (compared with the groups, as determined by colony formation assay). (C) Roxyl-ZR dramatically suppressed the proliferation of UM1 and TSCCA cells by decreasing the ratio of BrdU ${ }^{+}$cells, as determined by APC-BrdU staining. 


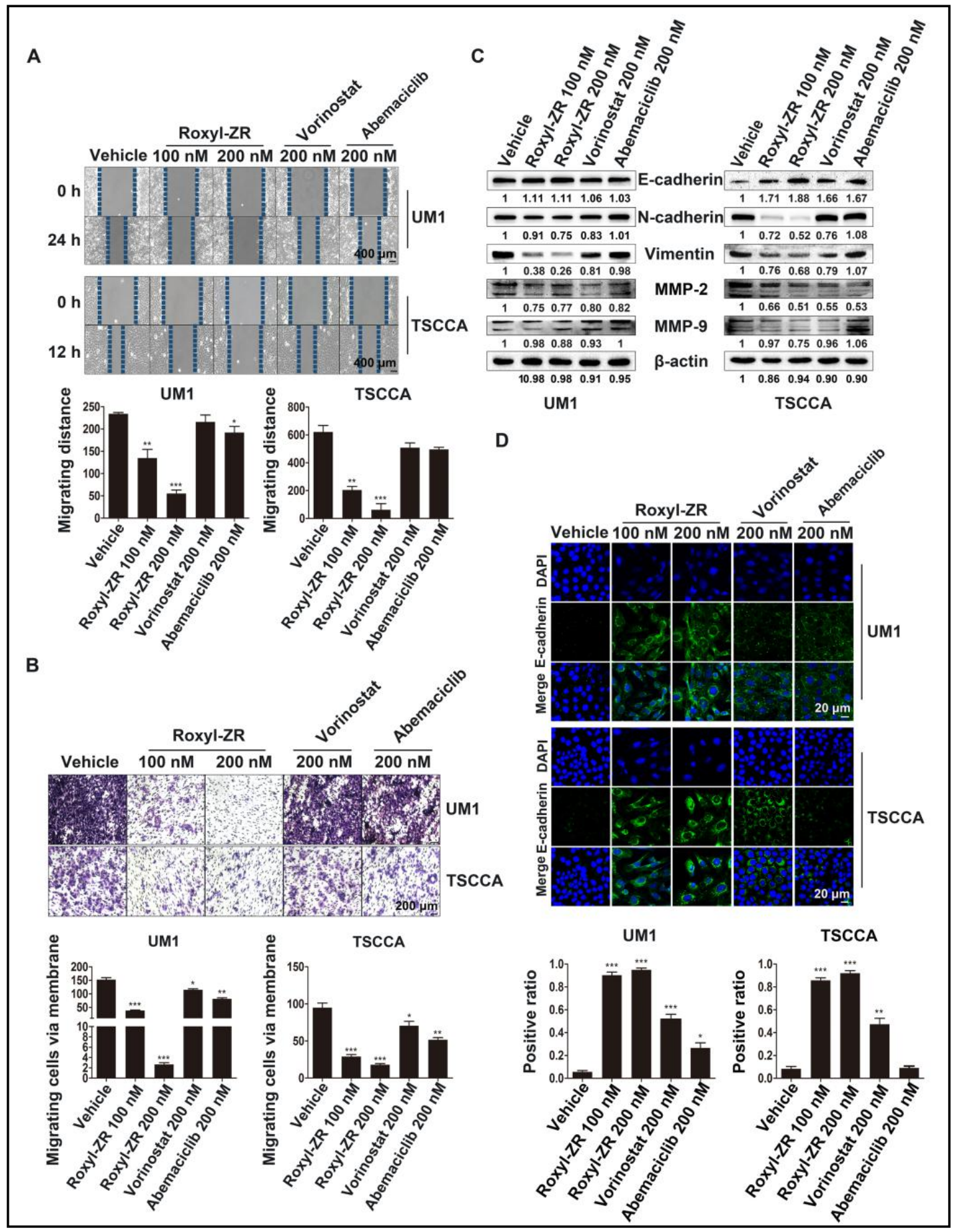

Fig. 3. Roxyl-ZR inhibits migration and invasion of OSCC cells. (A) Roxyl-ZR delayed scratch healing in UM1 and TSCCA cells (compared with other groups, as determined by a wound healing assay). (B) Roxyl-ZR effectively decreased the invasion of UM1 and TSCCA cells compared with the other groups, as determined by Transwell assay. (C) Cells were treated with Roxyl-ZR, vorinostat, abemaciclib, or vehicle for $48 \mathrm{~h}$. RoxylZR significantly up-regulated E-cadherin abundance and down-regulated N-cadherin, vimentin, MMP-2, and MMP-9 abundance with anti- $\beta$-actin as a loading control, as determined by western blot assay. (D) Roxyl-ZR significantly induced E-cadherin expression compared with other groups, as determined by immunofluorescence assay. E-cadherin in green was stained by Alexa-488 and cell nuclei were stained by DAPI (blue). 


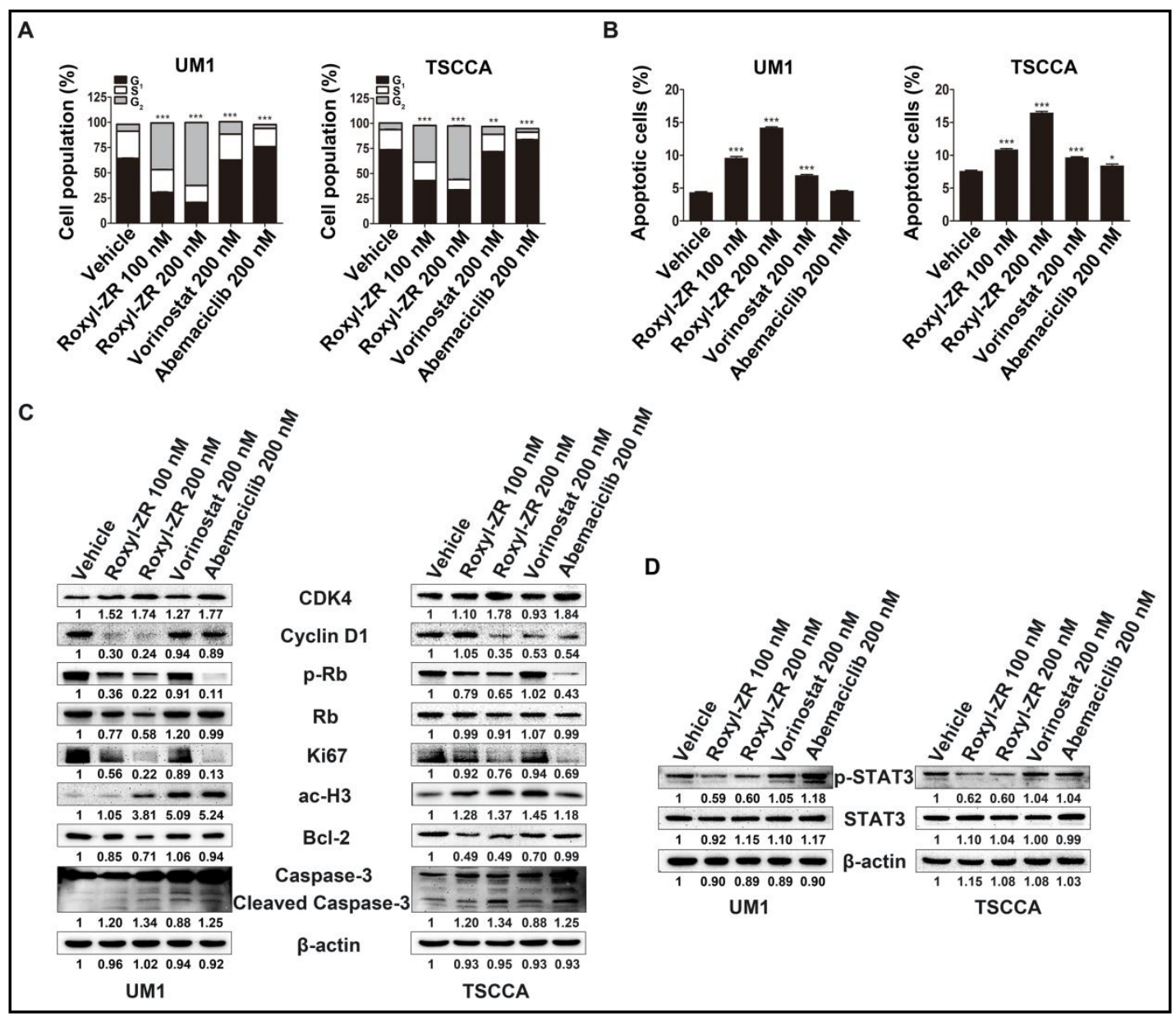

Fig. 4. (A) Cell cycle arrest analysis indicated that Roxyl-ZR induced $G_{2}$ arrest accompanied by reduction of $G_{0} G_{1}$ and the $S$ phase, as determined by FACS. (B) Apoptosis analysis showed that Roxyl-ZR mediated an increase in apoptosis compared to other groups by AV/PI dual staining. (C) After cells were treated with Roxyl-ZR, vorinostat, abemaciclib, or vehicle, the levels of cyclin D1, p-Rb, Ki67, and Bcl-2 abundance were significantly down-regulated, while those of CDK4, ac-H3, and cleaved caspase-3 abundance were up-regulated with anti- $\beta$-actin as a loading control, as determined by western blot assay. (D) Roxyl-ZR decreased the level of p-STAT3 abundance in a dose-dependent manner with anti- $\beta$-actin as a loading control, as determined by western blot assay.

STAT3 pathway may be responsible for the sensitization of OSCC to HDACis, which suggests that combined inhibition of HDAC with CDK is a potential therapy for OSCC.

\section{Roxyl-ZR inhibited tumor growth and invasion in vivo}

To better assess whether Roxyl-ZR could efficiently restrain tumor growth in vivo, two types of nude mice xenograft tumor models were established using UM1 or UM1-luc cells labeled with a reporter system encoding firefly luciferase by lentiviral transduction. As OSCC is highly correlated with smoking, drinking alcohol, and chewing betel quid, the incident of OSCC is more prevalent in males than in females. Thus, we used male nude mice in the animal experiments. For subcutaneous tumor models, tumor growth curves demonstrated that Roxyl-ZR treatments were effective compared with the treatments of the other abundance groups, especially Roxyl-ZR 40 and $80 \mathrm{mg} / \mathrm{kg}$ (Fig. 5A). There were minimal changes among Roxyl-ZR groups, while in the vorinostat or abemaciclib groups, the body weights of the mice decreased slightly (Fig. 5B). An obvious decrease in tumor size was also observed at the end-point of the study (Fig. 5C). Immunohistochemistry (IHC) staining showed that the 


\section{A}

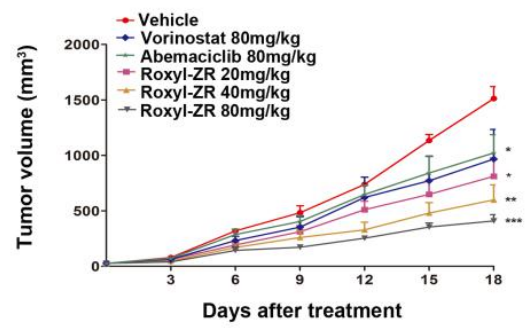

C

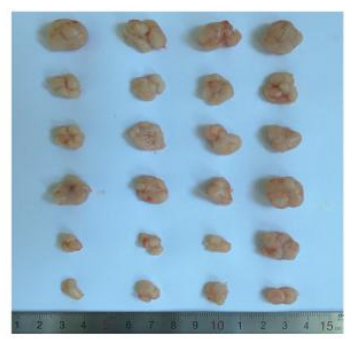

Vehicle

Vorinostat $80 \mathrm{mg} / \mathrm{kg}$

Abemaciclib $80 \mathrm{mg} / \mathrm{kg}$

Roxyl-ZR 20mg/kg

Roxyl-ZR 40mg/kg

Roxyl-ZR 80mg/kg

D

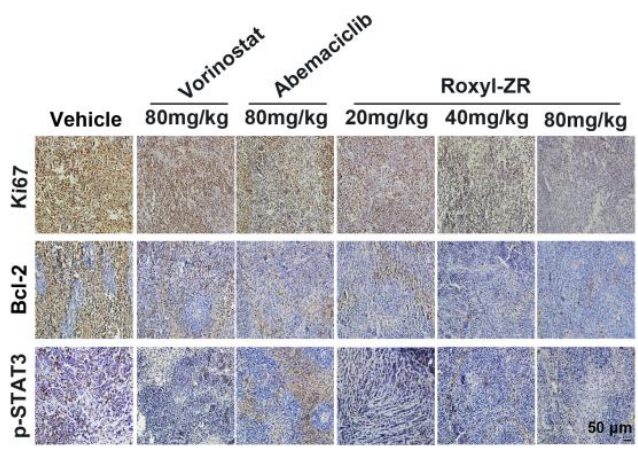

E

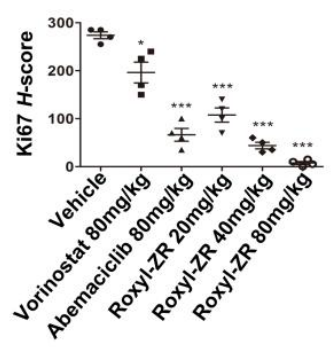

B

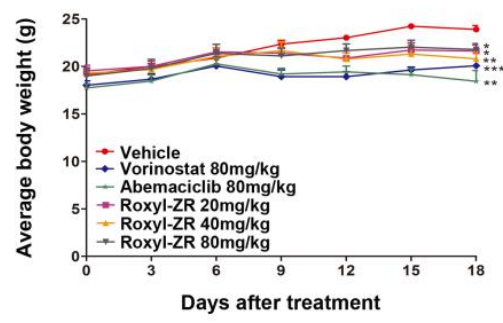

$\mathbf{F}$
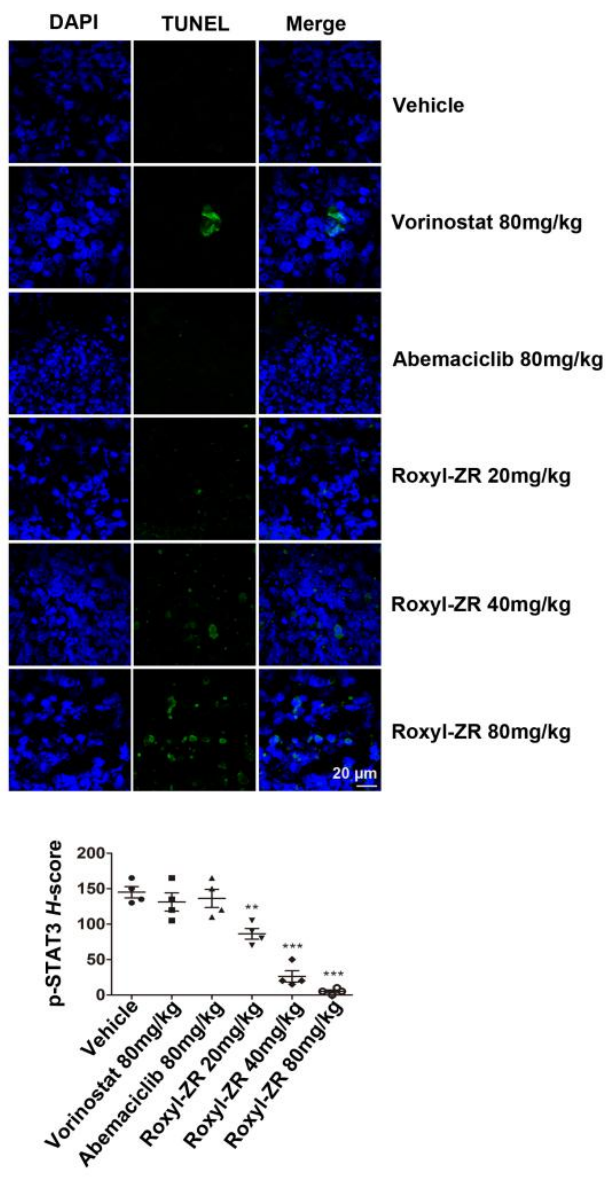

Fig. 5. Roxyl-ZR restrains UM1 subcutaneous xenograft tumor growth. (A) Growth curves of Roxyl-ZR $(20 \mathrm{mg} / \mathrm{kg}, 40 \mathrm{mg} / \mathrm{kg}, 80 \mathrm{mg} / \mathrm{kg})-$, vorinostat (80 mg/kg)-, abemaciclib (80 mg/kg)-, or vehicle-treated subcutaneous xenograft tumors. The growth rate in the Roxyl-ZR groups was inhibited compared with the other groups at day 18. (B) Average body weights of xenograft tumor mice after treatment with RoxylZR, vorinostat, abemaciclib, or vehicle. (C) Photograph of excised tumors with respective mice at day 18. Treatment with Roxyl-ZR resulted in a decrease in tumor volume compared with other groups. (D and E) Levels of Ki67, Bcl-2, and p-STAT3 expression were down-regulated in Roxyl-ZR groups in a dose-dependent manner compared with the other groups, as determined by IHC staining. (F) TUNEL assay indicated dosedependent apoptotic nuclei (green) in Roxyl-ZR groups.

expression levels of Ki67, Bcl-2, and p-STAT3 were suppressed among Roxyl-ZR groups in a dose-dependent manner (Fig. 5D and 5E). A TUNEL assay illustrated the increased level of apoptosis in Roxyl-ZR groups compared to other groups (Fig. 5F).

For orthotopic tumor models, BLI was used to evaluate tumor volume and density after the different treatments for 28 days. As shown in Fig. $6 \mathrm{~A}$ and 6B, photons emitted from 


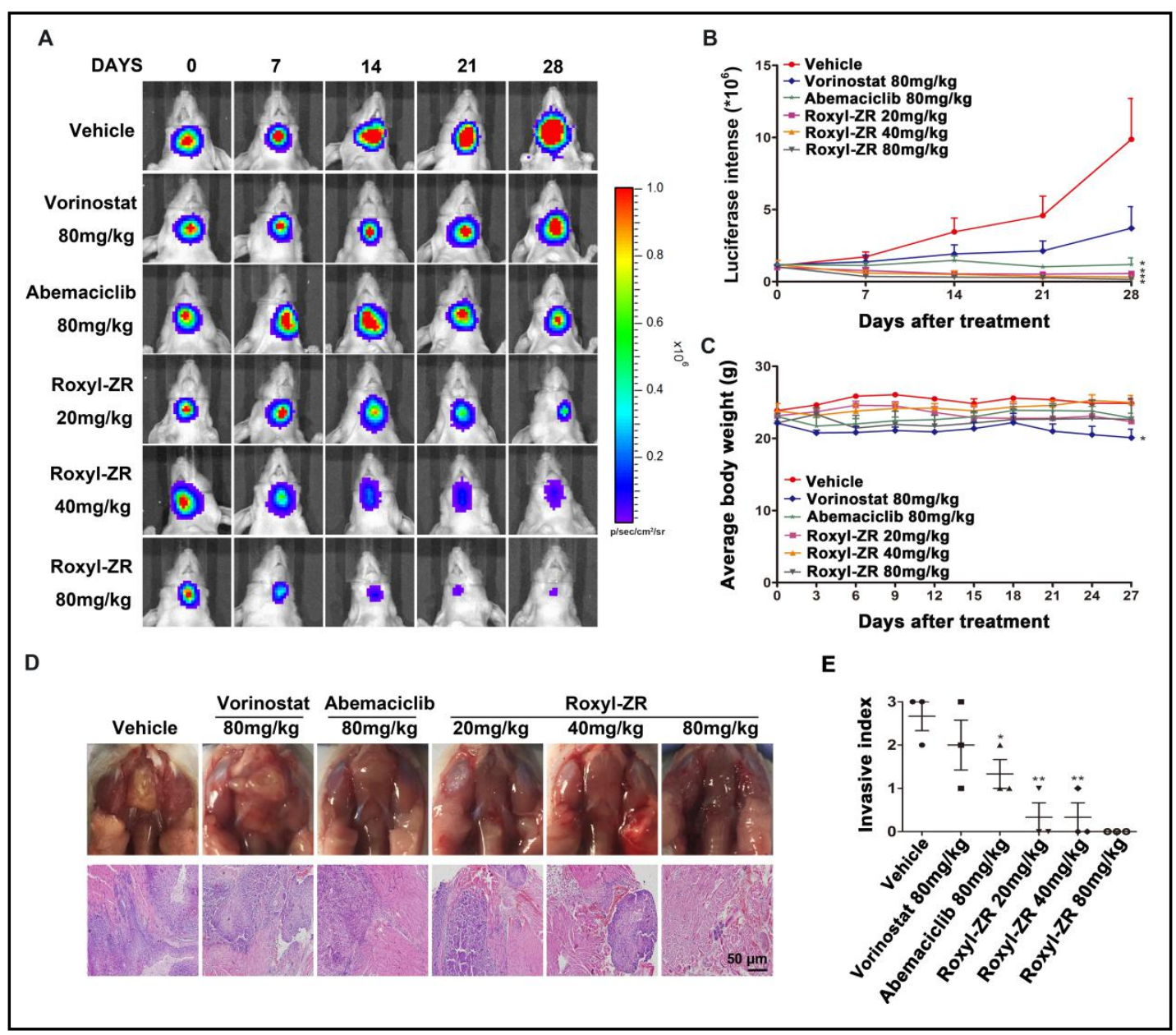

Fig. 6. Roxyl-ZR suppresses UM1-luc orthotopic xenograft tumor growth and invasion. (A) Tumor growth was monitored by BLI every 7 days. Tumors treated with Roxyl-ZR were significantly inhibited compared with the other groups. (B) Growth curves of orthotopic tumors treated with Roxyl-ZR, vorinostat, abemaciclib, or vehicle. The regions of -interest of tumors as shown in (A) were measured. (C) Average body weights of xenograft tumor mice after treatment with Roxyl-ZR, vorinostat, abemaciclib, or vehicle. (D and E) Photographs of orthotopic tumors from the different groups. Roxyl-ZR prevented orthotopic tumors from invading into the adjacent muscle layers, as determined by HE staining.

orthotopic tumors were markedly reduced among the Roxyl-ZR groups compared with the other groups. As the tumors in the floor of the mouth grew, the mice had difficulty eating, which resulted in weight loss. Other than the vorinostat group, none of the groups showed obvious abundance changes in average body weight, which indicated that gavage with Roxyl-ZR was effective and had low toxicity (Fig. 6C). The photographs of local incisions of the floor of the mouth illustrated that tumors of the Roxyl-ZR groups were smaller and the muscles had better morphological integrity compared with the other groups. However, in the vorinostat and vehicle groups, tumors adhered to the adjacent masseter muscle. Masseter muscle invasion was detected in the vorinostat and vehicle groups by HE staining, while in the Roxyl-ZR group, the tumors had sharp borders against adjacent muscle tissues (Fig. 6D and 6E). These observations indicated that Roxyl-ZR inhibited both tumor growth and invasiveness in subcutaneous and orthotopic tumor models, and that the effect was not attributable to systemic toxicity. 


\section{Cellular Physiology Cell Physiol Biochem 2019;53:141-156 \\ \begin{tabular}{ll|l} 
and Biochemistry $\begin{array}{l}\text { DOl: 10.33594/000000126 } \\
\text { Published online: } 26 \text { June 2019 }\end{array}$ & $\begin{array}{l}\text { O } 2019 \text { The Author(s). Published by } \\
\text { Cell Physiol Biochem Press GmbH\&Co. KG }\end{array}$ \\
\cline { 2 - 3 } &
\end{tabular} \\ Zhao et al.: Novel CDK-HDAC Inhibitor for the Treatment of Oral Cancer}

\section{Discussion}

Neoadjuvant chemotherapy has been investigated over a long period with the aim of reducing surgical margins, metastasis rates, and improving outcomes in different types of cancer, especially OSCC [27]. However, the therapeutic effects of current regimens are unsatisfactory due to the high rate of recurrences and multiple side effects $[28,29]$. Multitarget inhibitors have yielded superior efficacy compared with traditional agents due to the simultaneous targeting of multiple pathways critical for tumor growth. Combining multiple drugs that work through different mechanisms has been proven to be a therapeutic strategy for cancer management. Research has implied that the currently available HDACis could synergistically exert important antitumor activities as part of a combination therapy and permit lowering of the doses needed for adequate inhibition of tumor growth. To this end, the novel inhibitor Roxyl-ZR based on HDAC synergy against OSCC was developed in this study. Roxyl-ZR remarkably enhanced antitumor activity in OSCC both in in vitro and in vivo assays.

Functional assays revealed that Roxyl-ZR ultimately inhibited various processes in OSCC cells in vitro, including metabolism, clonogenicity, proliferation, migration, and invasion. Roxyl-ZR was more potent than control groups in these tests. Roxyl-ZR significantly induced $\mathrm{G}_{2}$ cell cycle arrest and apoptosis in OSCC cells. Western blot analysis demonstrated that RoxylZR exerted its antitumor functions by directly targeting HDAC and CDK and antagonizing downstream signaling cascades. In addition, Roxyl-ZR reduced the abundance of the antiapoptotic proteins Bcl-2 and enhanced the abundance of cleaved caspase- 3 .

In in vivo assays, Roxyl-ZR showed excellent antitumor effects in both subcutaneous and orthotopic tumor models and no reductions in body weight. Among the orthotopic tumor mice, HE staining demonstrated that oral administration with Roxyl-ZR prevented tumors from invading into adjacent muscle tissues. Importantly, compound Roxyl-ZR was more effective than the HDACi and CDK inhibitor, and even the HDAC plus CDK inhibitors in mice models. Therefore, Roxyl-ZR, which simultaneously targeted CDK and HDAC, enhanced the therapeutic effect of an HDACi.

The JAK-STAT signaling pathway has been implicated in malignant transformation and in tumor cell survival, invasion, and metastasis [30-33]. In a previous study, STAT3 stimulated anti-apoptotic signaling accounted for the limited response to HDACi treatment [2]. In the present study, the abundance level of p-STAT3 signaling with downstream transcriptional factors Bcl-2, Cyclin D1, MMP-2 and MMP-9 were restrained by Roxyl-ZR. As a result, RoxylZR inhibited metabolism, clonogenicity, proliferation, migration, and invasion of OSCC cells through reduced JAK1-STAT3 signaling in vitro and in vivo. STAT3 signaling confers the synergy of HDAC and CDK, but further evidence is needed. An in-depth mechanistic study of the conjugate compounds is ongoing in our laboratory and will be reported in due course.

\section{Conclusion}

In conclusion, a series of in vitro and in vivo studies provided evidence for the application of a pharmacophore merging strategy combining a HDACi with a CDK inhibitor displaying synergistic activities for the treatment of OSCC. Concurrent treatment by targeting CDK enhances OSCC cell sensitivity of HDACis. It is still possible to develop fabricate carrier-free HDACi/CDK inhibitor nanoparticles [34, 35]. The HDAC-CDK conjugates represent a novel approach for the development of OSCC therapy. Our study may open a new avenue for the development of novel inhibitors for the treatment of OSCC. 


\section{Cellular Physiology Cell Physiol Biochem 2019;53:141-156

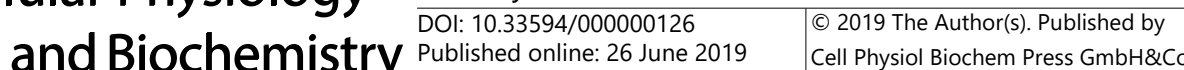 \\ Zhao et al.: Novel CDK-HDAC Inhibitor for the Treatment of Oral Cancer}

\section{Abbreviations}

HDACi (histone deacetylase inhibitor); OSCC (oral squamous cell carcinoma); CDKs (cyclin-dependent kinases); pLV-Luc (pLv-EF1 $\alpha$-luciferase-IRES-Neo); UM1-luc (UM1 cells with luciferase expression); HE (Hematoxylin and eosin); BLI (Bioluminescent Imaging); ROIs (Regions of Interest).

\section{Acknowledgements}

This work was supported by the Project of Science and Technology Assistance in Developing Countries (KY201501006) and the National Natural Science Foundation of China (81470354) and the Natural Science Foundation of Tianjin (17JCQNJC13500).

\section{Disclosure Statement}

The authors have no conflicts of interest to disclose.

\section{References}

1 Zhang L, Sun ZJ, Bian Y, Kulkarni AB: MicroRNA-135b acts as a tumor promoter by targeting the hypoxiainducible factor pathway in genetically defined mouse model of head and neck squamous cell carcinoma. Cancer Lett 2013;331:230-238.

2 Feller L, Lemmer J: Oral squamous cell carcinoma: Epidemiology, clinical presentation and treatment. J Cancer Ther 2012;3:263-268.

3 Specenier PM, Vermorken JB: Current concepts for the management of head and neck cancer: chemotherapy. Oral Oncol 2009;45:409-415.

4 Patel V, Senderowicz AM, Pinto D, Jr., Igishi T, Raffeld M, Quintanilla-Martinez L, Ensley JF, Sausville EA, Gutkind JS: Flavopiridol, a novel cyclin-dependent kinase inhibitor, suppresses the growth of head and neck squamous cell carcinomas by inducing apoptosis. J Clin Invest 1998;102:1674-1681.

5 Peng M, Darko KO, Tao T, Huang Y, Su Q, He C, Yin T, Liu Z, Yang X: Combination of metformin with chemotherapeutic drugs via different molecular mechanisms. Cancer Treat Rev 2017;54:24-33.

6 Barzegar M, Ma S, Zhang C, Chen X, Gu Y, Shang C, Jiang X, Yang J, Nathan CA, Yang S, Huang S: SKLB188 inhibits the growth of head and neck squamous cell carcinoma by suppressing EGFR signalling. Br J Cancer 2017;117:1154-1163.

7 Zhou TT, Quan LL, Chen LP, Du T, Sun KX, Zhang JC, Yu L, Li Y, Wan P, Chen LL, Jiang BH, Hu LH, Chen J, Shen X: SP6616 as a new Kv2.1 channel inhibitor efficiently promotes beta-cell survival involving both PKC/ Erk1/2 and CaM/PI3K/Akt signaling pathways. Cell Death Dis 2016;7:e2216.

8 Wu DW, Chen TC, Huang HS, Lee H: TC-N19, a novel dual inhibitor of EGFR and cMET, efficiently overcomes EGFR-TKI resistance in non-small-cell lung cancer cells. Cell Death Dis 2016;7:e2290.

9 Hartwell LH: Saccharomyces cerevisiae cell cycle. Bacteriol Rev 1974;38:164-198.

10 Mueller S, Yang X, Sottero TL, Gragg A, Prasad G, Polley MY, Weiss WA, Matthay KK, Davidoff AM, DuBois SG, Haas-Kogan DA: Cooperation of the HDAC inhibitor vorinostat and radiation in metastatic neuroblastoma: efficacy and underlying mechanisms. Cancer Lett 2011;306:223-229.

11 Khan 0, La Thangue NB: HDAC inhibitors in cancer biology: emerging mechanisms and clinical applications. Immunol Cell Biol 2012;90:85-94.

12 Barneda-Zahonero B, Parra M: Histone deacetylases and cancer. Mol Oncol 2012;6:579-589.

13 Tasoulas J, Giaginis C, Patsouris E, Manolis E, Theocharis S: Histone deacetylase inhibitors in oral squamous cell carcinoma treatment. Expert Opin Investig Drugs 2015;24:69-78. 


\section{Cellular Physiology Cell Physiol Biochem 2019;53:141-156

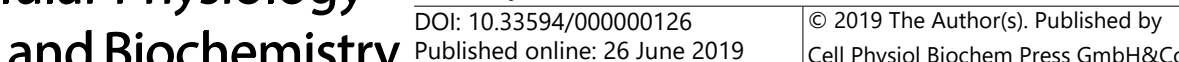

14 Haigentz M, Jr., Kim M, Sarta C, Lin J, Keresztes RS, Culliney B, Gaba AG, Smith RV, Shapiro GI, Chirieac LR, Mariadason JM, Belbin TJ, Greally JM, Wright JJ, Haddad RI: Phase II trial of the histone deacetylase inhibitor romidepsin in patients with recurrent/metastatic head and neck cancer. Oral Oncol 2012;48:1281-1288.

15 Suzuki M, Endo M, Shinohara F, Echigo S, Rikiishi H: Enhancement of cisplatin cytotoxicity by SAHA involves endoplasmic reticulum stress-mediated apoptosis in oral squamous cell carcinoma cells. Cancer Chemother Pharmacol 2009;64:1115-1122.

16 Shen J, Huang C, Jiang L, Gao F, Wang Z, Zhang Y, Bai J, Zhou H, Chen Q: Enhancement of cisplatin induced apoptosis by suberoylanilide hydroxamic acid in human oral squamous cell carcinoma cell lines. Biochem Pharmacol 2007;73:1901-1909.

17 Dawson MA, Kouzarides T, Huntly BJ: Targeting epigenetic readers in cancer. N Engl J Med 2012;367:647657.

18 Minucci S, Pelicci PG: Histone deacetylase inhibitors and the promise of epigenetic (and more) treatments for cancer. Nat Rev Cancer 2006;6:38-51.

19 Li Y, Guo Q Zhang C, Huang Z, Wang T, Wang X, Wang X, Xu G, Liu Y, Yang S, Fan Y, Xiang R: Discovery of a highly potent, selective and novel CDK9 inhibitor as an anticancer drug candidate. Bioorg Med Chem Lett 2017;27:3231-3237.

20 Li Y, Luo X, Guo Q, Nie Y, Wang T, Zhang C, Huang Z, Wang X, Liu Y, Chen Y, Zheng J, Yang S, Fan Y, Xiang R: Discovery of N1-(4-((7-Cyclopentyl-6-(dimethylcarbamoyl)-7 H-pyrrolo [2, 3- d]pyrimidin-2-yl)amino) phenyl)- N8-hydroxyoctanediamide as a Novel Inhibitor Targeting Cyclin-dependent Kinase 4/9 (CDK4/9) and Histone Deacetlyase1 (HDAC1) against Malignant Cancer. J Med Chem 2018;61:3166-3192.

21 Yang S, Zhao L, Yang J, Chai D, Zhang M, Zhang J, Ji X, Zhu T: deltaEF1 represses BMP-2-induced differentiation of C2C12 myoblasts into the osteoblast lineage. J Biomed Sci 2007;14:663-679.

22 Liu Z, Tong Y, Liu Y, Liu H, Li C, Zhao Y, Zhang Y: Effects of suberoylanilide hydroxamic acid (SAHA) combined with paclitaxel (PTX) on paclitaxel-resistant ovarian cancer cells and insights into the underlying mechanisms. Cancer Cell Int 2014;14:112.

23 He JC, Yao W, Wang JM, Schemmer P, Yang Y, Liu Y, Qian YW, Qi WP, Zhang J, Shen Q Yang T: TACC3 overexpression in cholangiocarcinoma correlates with poor prognosis and is a potential anti-cancer molecular drug target for HDAC inhibitors. Oncotarget 2016;7:75441-75456.

24 Brehm A, Miska EA, McCance DJ, Reid JL, Bannister AJ, Kouzarides T: Retinoblastoma protein recruits histone deacetylase to repress transcription. Nature 1998;391:597-601.

25 Harbour JW, Dean DC: The Rb/E2F pathway: expanding roles and emerging paradigms. Genes Dev 2000;14:2393-2409.

26 Zeng H, Qu J, Jin N, Xu J, Lin C, Chen Y, Yang X, He X, Tang S, Lan X, Yang X, Chen Z, Huang M, Ding J, Geng M: Feedback Activation of Leukemia Inhibitory Factor Receptor Limits Response to Histone Deacetylase Inhibitors in Breast Cancer. Cancer Cell 2016;30:459-473.

27 Diyabalanage HV, Granda ML, Hooker JM: Combination therapy: histone deacetylase inhibitors and platinum-based chemotherapeutics for cancer. Cancer Lett 2013;329:1-8.

28 Vishak S, Rangarajan B, Kekatpure VD: Neoadjuvant chemotherapy in oral cancers: Selecting the right patients. Indian J Med Paediatr Oncol 2015;36:148-153.

29 Tabrizi R, Garajei A, Shafie E, Jamshidi S: Outcome of Neoadjuvant Chemotherapy on Local Recurrence and Distant Metastasis of Oral Squamous Cell Carcinoma: A Retrospective Study. J Dent (Shiraz) 2016;17:207212.

30 Kong Y, Li F, Nian Y, Zhou Z, Yang R, Qiu MH, Chen C: KHF16 is a Leading Structure from Cimicifuga foetida that Suppresses Breast Cancer Partially by Inhibiting the NF-kappaB Signaling Pathway. Theranostics 2016;6:875-886.

31 Siveen KS, Nguyen AH, Lee JH, Li F, Singh SS, Kumar AP, Low G, Jha S, Tergaonkar V, Ahn KS, Sethi G: Negative regulation of signal transducer and activator of transcription-3 signalling cascade by lupeol inhibits growth and induces apoptosis in hepatocellular carcinoma cells. Br J Cancer 2014;111:1327-1337.

32 Zhong Z, Wen Z, Darnell JE, Jr:: Stat3: a STAT family member activated by tyrosine phosphorylation in response to epidermal growth factor and interleukin-6. Science 1994;264:95-98.

33 Hsu FT, Chang B, Chen JC, Chiang IT, Liu YC, Kwang WK, Hwang JJ: Synergistic Effect of Sorafenib and Radiation on Human Oral Carcinoma in vivo. Sci Rep 2015;5:15391. 


\section{Cellular Physiology Cell Physiol Biochem 2019;53:141-156}

\begin{tabular}{ll|l} 
and Biochemistry & $\begin{array}{l}\text { DOl: 10.33594/000000126 } \\
\text { Published online: } 26 \text { June 2019 }\end{array}$ & $\begin{array}{l}\text { O } 2019 \text { The Author(s). Published by } \\
\text { Cell Physiol Biochem Press GmbH\&Co. KG }\end{array}$ \\
\cline { 2 - 3 } &
\end{tabular}

Zhao et al.: Novel CDK-HDAC Inhibitor for the Treatment of Oral Cancer

34 Chen F, Zhao Y, Pan Y, Xue X, Zhang X, Kumar A, Liang XJ: Synergistically Enhanced Therapeutic Effect of a Carrier-Free HCPT/DOX Nanodrug on Breast Cancer Cells through Improved Cellular Drug Accumulation. Mol Pharm 2015;12:2237-2244.

35 Zhao Y, Chen F, Pan Y, Li Z, Xue X, Okeke CI, Wang Y, Li C, Peng L, Wang PC, Ma X, Liang XJ: Nanodrug Formed by Coassembly of Dual Anticancer Drugs to Inhibit Cancer Cell Drug Resistance. ACS Appl Mater Interfaces 2015;7:19295-19305. 